\title{
Open
}

\section{MSH6 and PMS2 germ-line pathogenic variants implicated in Lynch syndrome are associated with breast cancer}

\author{
Maegan E. Roberts, $\mathrm{MS}^{1}$, Sarah A. Jackson, $\mathrm{MS}^{1}$, Lisa R. Susswein, MS, MHA ${ }^{1}$, \\ Nur Zeinomar, PhD, MPH${ }^{2}$, Xinran Ma, MS $^{2}$, Megan L. Marshall, MS ${ }^{1}$, Amy R. Stettner, MS ${ }^{1}$, \\ Becky Milewski, MS ${ }^{1}$, Zhixiong $\mathrm{Xu}, \mathrm{PhD}^{1}$, Benjamin D. Solomon, $\mathrm{MD}^{1}$, Mary Beth Terry, $\mathrm{PhD}^{2}$, \\ Kathleen S. Hruska, PhD ${ }^{1}$, Rachel T. Klein, $\mathrm{MS}^{1}$ and Wendy K. Chung, MD, PhD ${ }^{3}$
}

Purpose: An association of Lynch syndrome (LS) with breast cancer has been long suspected; however, there have been insufficient data to address this question for each of the LS genes individually.

Methods: We conducted a retrospective review of personal and family history in 423 women with pathogenic or likely pathogenic germ-line variants in MLH1 $(N=65), M S H 2(N=94)$, MSH6 $(N=140)$, or PMS2 $(N=124)$ identified via clinical multigene hereditary cancer testing. Standard incidence ratios (SIRs) of breast cancer were calculated by comparing breast cancer frequencies in our study population with those in the general population (Surveillance, Epidemiology, and End Results 18 data).

Results: When evaluating by gene, the age-standardized breast cancer risks for MSH6 (SIR = 2.11; 95\% confidence interval (CI), 1.56-2.86) and PMS2 (SIR $=2.92 ; 95 \% \mathrm{CI}, 2.17-3.92)$ were associated with a statistically significant risk for breast cancer whereas no association was observed for MLH1 (SIR = 0.87; 95\% CI, 0.42-1.83) or MSH2 (SIR $=1.22 ; 95 \%$ CI, 0.72-2.06).

Conclusion: Our data demonstrate that two LS genes, MSH6 and PMS2, are associated with an increased risk for breast cancer and should be considered when ordering genetic testing for individuals who have a personal and/or family history of breast cancer.

Genet Med advance online publication 18 January 2018

Key Words: breast cancer; Lynch syndrome; mismatch repair; MSH6; PMS2

identify patients with LS. Although screening for MMR deficiency in breast tumors is currently not standard clinical practice, multiple studies have shown that breast cancers from women with LS are more likely to exhibit microsatellite instability and loss of one or more MMR proteins via immunohistochemistry, compared with sporadic breast cancers. $^{3-14}$

Clinical studies evaluating breast cancer risk in women with LS have been conflicting, with some showing up to a fourfold increased breast cancer risk and others reporting no increased risk. ${ }^{15,16}$ Ascertainment bias may have affected results: many previous LS studies assessing breast cancer ascertained their study cohorts based on criteria heavily weighted toward colorectal cancer. As a result, many study cohorts consisted only of $M L H 1$ and $M S H 2$ carriers although some also included MSH6. ${ }^{17-23}$ A few studies have included all four MMR genes, but presented only a single combined breast cancer risk, likely due to a small number of MSH6 and PMS2 carriers. ${ }^{15,16}$ Thus, the lack of consistent association of breast cancer and LS in previous studies may reflect the genetic composition of the cohorts. While it has been suggested that breast cancer risk may vary by gene in LS, no one study has examined gene- and age-specific breast cancer risks for all four MMR genes. The aim of our study was to characterize

${ }^{1}$ GeneDx, Gaithersburg, Maryland, USA; ${ }^{2}$ Department of Epidemiology, Columbia University, New York, New York, USA; ${ }^{3}$ Departments of Pediatrics and Medicine, Columbia University, New York, New York, USA. Correspondence: Maegan E. Roberts (mroberts@genedx.com) 
breast cancer risks for each MMR gene in our group of women who were identified to have a PV via germ-line hereditary cancer panel testing for a variety of cancer-related indications.

\section{Participants}

We retrospectively queried more than 50,000 women who had multigene hereditary cancer panel testing completed at GeneDx between 2013 and 2016, identifying 423 women with a single germ-line pathogenic variant or likely pathogenic variant (collectively referred to here as PV) in any of the four MMR genes. All sequence variants were classified based on the 2015 American College of Medical Genetics and Genomics/Association for Molecular Pathology guidelines for the interpretation of sequence variants. ${ }^{24}$ Combined EPCAMMSH2 deletions were included in the MSH2 PV cohort. Individuals with a second PV in an MMR or another gene (excluding single PV in MUTYH) were excluded. A list of the multigene panels (with gene lists) included and PVs identified can be found in Supplementary Tables S1 and S2 online.

Demographic, clinical, and family history information was obtained from test requisition forms and accompanying clinical documents supplied by the ordering provider at the time of the test order. Pathology reports were not requested to confirm diagnoses. Subjects were limited to females over the age of 18. Breast cancer was defined as any invasive breast neoplasm or ductal carcinoma in situ. This study was approved by the Western Institutional Review Board, Puyallup, WA (WIRB 20162523).

\section{Technical/laboratory methods}

Genomic DNA was isolated from whole blood using a QIAsymphony DNA kit, and from oral rinse using a QIAsymphony DSP Virus/Pathogen Midi Kit (Qiagen, Valencia, CA). Genomic DNA was enriched for the complete coding region and splice-site junctions of the genes of interest using custom SureSelect targeted capture (Agilent, Santa Clara, CA). Next-generation sequencing and deletion/duplication analysis were performed for all coding regions as well as a portion of the $5^{\prime}$ untranslated region, $3^{\prime}$ untranslated region, and intronic regions for all genes on each panel, with the exception of EPCAM, for which only deletion/duplication analysis was performed. The products were sequenced on Illumina MiSeq or HiSeq instruments with paired-end reads (Illumina, San Diego, CA). DNA sequence was mapped to a masked version of the published human genome build University of California-Santa Cruz hg19/GRCh37 reference sequence using BWA-Mem version 0.7.8. ${ }^{25,26}$ Local realignment around insertion/deletion sites and regions with poor mapping quality was performed using the Genome Analysis Toolkit version 1.6 IndelRealigner. ${ }^{27}$ Variant calls were generated by the union of SAMtools version $0.1 .18,{ }^{28}$ Genome Analysis Toolkit UnifiedGenotyper, ${ }^{27}$ and a GeneDxdeveloped heuristic caller. Capillary sequencing (Applied Biosystems/Life Technologies, Grand Island, NY) on a newly extracted DNA sample was used to confirm all variants with clinical or uncertain significance and to fill in sequence for regions with fewer than 15 reads by next-generation sequencing. Any potential variant position with coverage of fewer than 50 reads was reviewed by analysts and analyzed by capillary sequencing if unclear. Long-range polymerase chain reaction was used to distinguish variants in PMS2 from those in the PMS2 pseudogene PMS2CL. Deletion/duplication analysis was performed via custom-designed exon-targeted array comparative genomic hybridization (Agilent). Confirmation of copy-number changes detected on array comparative genomic hybridization was performed by multiple ligation-dependent probe amplification, repeat microarray analysis, or quantitative polymerase chain reaction using the Universal ProbeLibrary (Roche, Indianapolis, IN).

\section{Statistical analysis}

Standardized incidence ratios (SIRs) for breast cancer were calculated for each gene as the number of observed cancers to the number of expected cancers based on breast cancer incidence in the general population. The expected number of cases was estimated for each 5-year age group from 20 to 85 as the time at risk multiplied by the reference population incidence rates. The population-based incidence rates were obtained using SEER ${ }^{\star}$ Stat statistical software from the Surveillance, Epidemiology, and End Results (SEER) 18 data, which includes 18 cancer registries representing $27.8 \%$ of the US population. ${ }^{29,30}$ Corresponding $95 \%$ confidence intervals (95\% CI) were calculated using the Poisson approximation distribution. SIRs for endometrial, colorectal, and ovarian cancer were also calculated using their respective incidence rates from SEER 18. All statistical analyses were conducted using RStudio 0.99.902. ${ }^{31}$ The Kaplan-Meier method was used to estimate cumulative risk and corresponding 95\% CIs for breast cancers to age 60 , stratified by MMR gene. ${ }^{32}$ Logrank test was used to compare incidence rates between groups. All reported $p$ values are two-tailed with a significance level of 0.05 .

\section{RESULTS}

\section{Cohort characteristics}

Most of the 423 female MMR PV carriers in our study reported Caucasian or European ancestry $(72.1 \%, 305 / 423)$. The average age at time of genetic testing was $52.5( \pm 12.1)$ years (see Table 1 for further demographic details). The distribution of MMR PVs was as follows: MSH6, 33.1\% (140/423); PMS2, 29.3\% (124/423); MSH2, 22.2\% (94/423); MLH1, 15.4\% (65/423). The 423 women in our study harbored 241 unique PVs, the majority of which $(87.5 \%$, 370/423) were predicted to result in a truncated protein or a transcript subject to nonsense-mediated decay (start codon, splice, cryptic splice, nonsense, deletion/duplication, frameshift), while the rest were missense or small in-frame deletions $(12.5 \%, 53 / 423)$ (Supplementary Figure S1). 
Table 1 Demographic and clinical characteristics of study cohort

\begin{tabular}{lc}
\hline $\begin{array}{l}\text { Demographic/Clinical Characteristic } \\
\text { MMR PV Positive } \\
N=\mathbf{4 2 3}\end{array}$ \\
\hline MMR gene, No. (\%) \\
\hline MLH1 & $65(15.4)$ \\
\hline MSH2 & $94(22.2)$ \\
\hline MSH6 & $140(33.1)$ \\
\hline PMS2 & $124(29.3)$ \\
\hline Personal History & $107(25.3)$ \\
\hline Personal Hx of Breast Cancer, No. (\%) & $50.2(11.7)$ \\
\hline Average Age at Breast Cancer Dx, year (SD) & $26-76$ \\
\hline Range of Ages at Breast Cancer Dx, years & $52.5(12.1)$ \\
\hline Average Age at Testing, years (SD) & \\
\hline Self-reported Race/Ethnicity, No. (\%) & $325(83.8)$ \\
\hline Caucasian/European & $28(7.2)$ \\
\hline Hispanic & $24(6.2)$ \\
\hline Black/African American & $21(5.4)$ \\
\hline Asian/Pacific Islander & $8(2.1)$ \\
\hline Native American & $5(1.3)$ \\
\hline Other
\end{tabular}

No. = number; SD = standard deviation.

\#Percentages represent proportion of women who provided race/ethnicity information $(N=388)$. Women reporting more than one race/ethnicity are counted more than once.

\section{Cancer history and referral patterns}

A personal history of breast cancer was reported in $25.3 \%$ (107/423) of the cohort, with $1.4 \%(6 / 423)$ reporting a history of more than one primary breast cancer. The average age at first breast cancer diagnosis was 50.2 years $( \pm 11.7$, range: 26-76).

Approximately half of the women with a MMR PV were referred for testing with either the Comprehensive Cancer Panel $(24.6 \%, 104 / 423)$ or the Breast/Ovarian Cancer Panel $(24.3 \%, 103 / 423)$, both of which contain genes related to breast cancer and LS (Supplementary Table S1). Twenty-six percent (27/104) of Comprehensive Cancer and 45.6\% (47/103) of Breast/Ovarian Cancer Panel patients reported a personal history of breast cancer. Twenty-eight percent (117/423) of the women with MMR PVs were referred for the Colorectal Cancer Panel or the Lynch Syndrome Panel. Of these 117 women, 2 (1.7\%) had breast cancer. Reflex testing (more extensive genetic testing after the first panel yielded normal results) identified PVs in three women with breast cancer following a normal result on the High-Risk Breast Cancer Panel.

Prevalence of breast, ovarian, endometrial, and colorectal cancer varied among MLH1, MSH2, MSH6, and PMS2 carriers (Figure 1a). After preliminary analyses, genes were grouped based on similar breast cancer prevalence observed in MMR PV carriers ( $M L H 1 / M S H 2$ vs. MSH6/PMS2). A diagnosis of breast cancer, irrespective of other personal cancer history, was reported more frequently in women with MSH6 or PMS2 PVs (MSH6: 30\%, 42/140; PMS2: 35.5\%, 44/124) compared to women with $M L H 1$ or MSH2 PVs
(MLH1: $10.8 \%, 7 / 65 ;$ MSH2: $14.9 \%, 14 / 94)(p<0.001)$ (Figure 1a). Furthermore, breast cancer in the absence of LS-associated cancers was reported more frequently in women with PVs in MSH6 or PMS2 (MSH6: 18.6\%, 26/140; PMS2: $29.0 \%, 36 / 124)$ compared to women with PVs in MLH1 or MSH2 (MLH1: 3.1\%, 2/65; MSH2: 4.3\%, 4/94) $(p<0.001)$ (Figure 1b).

The average numbers of reported breast, ovarian, endometrial, and colorectal cancers per family showed similar results to personal cancer histories (Figure 1c). Breast cancer was more commonly reported in family members (up to third degree as available) of probands with a PV in MSH6 or PMS2 (average number of reported cancers per family $=1.46$ and 1.52) whereas colon cancer was more commonly reported in family members of probands with a PV in MLH1 or MSH2 (2.62 and 2.12). The proportion of probands (excluding the seven relatives in our cohort) reporting a personal or family history of breast cancer but no colorectal, endometrial, or ovarian cancer was lower among those with $M L H 1$ or $M S H 2$ PVs (MLH1: 0\%, 0/65; MSH2: 2.2\%, 2/92) than among those with PVs in MSH6 or PMS2 (MSH6: 8.0\%, 11/137; PMS2: $24.6 \%, 30 / 122)(p<0.001)$.

\section{SIR analysis and cumulative risk}

Breast cancer risk was found to be approximately twofold higher $(\mathrm{SIR}=1.96 ; 95 \% \mathrm{CI}, 1.63-2.37)$ in our aggregate mismatch repair PV cohort compared to the general population (Table 2). When evaluating by gene, only two of the four MMR genes were found to have a statistically significant increased association with breast cancer: MSH6 $(\mathrm{SIR}=2.11 ; 95 \% \mathrm{CI}, 1.56-2.86)$ and PMS2 (SIR $=2.92 ; 95 \%$ CI, 2.17-3.92). MLH1 (SIR =0.87; 95\% CI, 0.416-1.83) and MSH2 (SIR $=1.22 ; 95 \% \mathrm{CI}, 0.721-2.06)$ were not found to be associated with breast cancer.

Cumulative incidence of breast cancer was calculated and illustrated via Kaplan-Meier analysis (Figure 2). The cumulative incidence of breast cancer at the age of 60 was found to be $37.7 \%$ (95\% CI, 27.5-47.8) for PMS2, 31.1\% (95\% CI, 21.9-40.7) for MSH6, 16.1\% (95\% CI, 7.3-27.9) for MSH2, and $15.5 \%$ (95\% CI, 5.5-30.2) for MLH1 PV carriers. MSH6 and PMS2 had a statistically significant greater cumulative incidence of breast cancer compared with $\mathrm{MLH} 1$ and $\mathrm{MSH} 2$ $(p<0.001)$.

\section{Clinical criteria}

Unrelated probands $(n=416)$ were evaluated to determine if they met Amsterdam II, Revised Bethesda Guidelines, and the National Comprehensive Cancer Network (NCCN) BRCA1/2 Testing Criteria (Figure 3). ${ }^{33-35}$ Women with MLH1 (53.8\%, 35/65) or MSH2 (43.5\%, 40/92) PVs, compared with MSH6 and PMS2, were more likely to meet Amsterdam II criteria. Women with MLH1 PVs most frequently met Revised Bethesda Guidelines $(50.8 \%, 33 / 65)$. A higher proportion of women with MSH6 (64.2\%, 88/137) or PMS2 (68.9\%, 84/122) PVs met NCCN BRCA1/2 testing criteria compared to those with MLH1 (44.6\%, 29/65) or MSH2 (45.7\%, 42/92) PVs. 

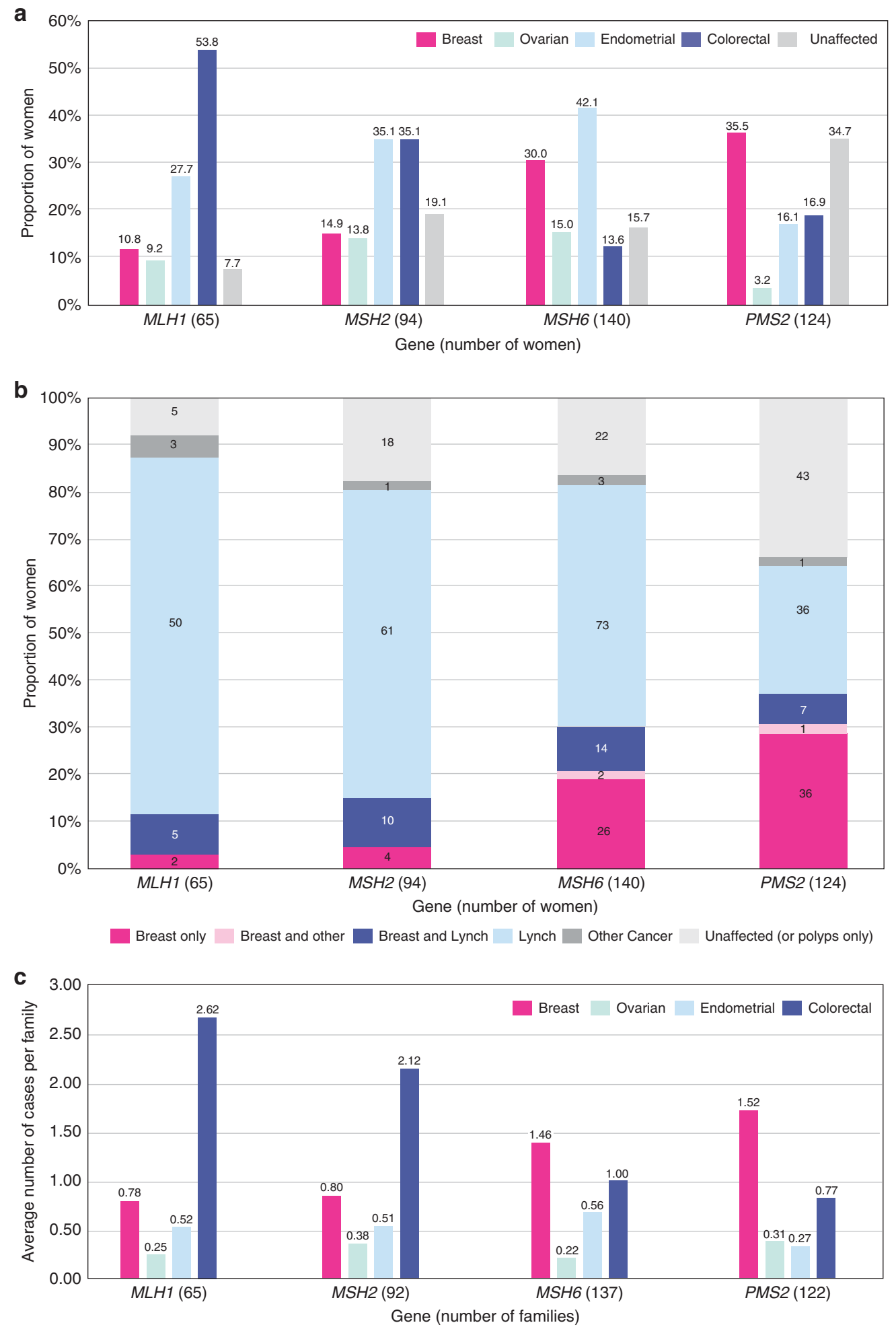

Figure 1 Personal and family history. (a) Personal history of breast and Lynch syndrome (LS)-associated cancers. Women with multiple cancers are counted more than once. Fallopian tube and primary peritoneal cancer were both included as ovarian cancer. Unaffected: no history of cancer but could have a history of colorectal polyps. (b) Personal history: breast cancer only versus other histories. Individuals are counted only once. Breast only: breast cancer, but no history of a LS-associated or any other cancer; Breast and Lynch: breast cancer plus a LS-associated tumor; Breast and other: breast cancer and possibly another non-LS-associated tumor; Lynch: LS-associated tumor and possibly other cancers; Other cancer: history of cancer that is not breast or a LS-associated tumor. (c) Family history: average number of cancer cases per family. Includes family history data only, not probands. Seven individuals were related to someone else in our cohort, but their families were only counted once. Total families $=416$. 
Table 2 Standard incidence ratios by MMR gene

\begin{tabular}{|c|c|c|c|c|c|}
\hline & $M L H 1\left(N=64^{\wedge}\right)$ & $M S H 2(N=94)$ & MSH6 $(N=140)$ & PMS2 $(N=124)$ & Total $(N=422)$ \\
\hline \multicolumn{6}{|l|}{ Breast Cancer } \\
\hline Observed, No. & 7 & 14 & 42 & 44 & 107 \\
\hline Expected, No. & 8.01 & 11.50 & 19.87 & 15.09 & 54.48 \\
\hline SIRs & 0.87 & 1.22 & 2.11 & 2.92 & 1.96 \\
\hline $95 \% \mathrm{Cl}$ & $(0.43-1.83)$ & $(0.72-2.06)$ & $(1.56-2.86)$ & $(2.17-3.92)$ & $(1.63-2.37)$ \\
\hline$p$ value & 0.72 & 0.46 & $<0.001$ & $<0.001$ & $<0.001$ \\
\hline Expected, No. & 1.74 & 2.49 & 6.01 & 4.30 & 14.54 \\
\hline SIRs & 19.53 & 13.25 & 3.16 & 4.65 & 7.29 \\
\hline $95 \% \mathrm{Cl}$ & $(13.96-27.34)$ & $(9.42-18.64)$ & $(2.02-4.96)$ & $(2.30-7.20)$ & $(6.03-8.82)$ \\
\hline$p$ value & $<0.001$ & $<0.001$ & $<0.001$ & $<0.001$ & $<0.001$ \\
\hline $95 \% \mathrm{Cl}$ & $(8.06-20.86)$ & $(12.66-25.32)$ & $(11.89-19.81)$ & $(4.36-10.47)$ & $(10.87-15.37)$ \\
\hline$p$ value & $<0.001$ & $<0.001$ & $<0.001$ & $<0.001$ & $<0.001$ \\
\hline \multicolumn{6}{|l|}{ Ovarian Cancer } \\
\hline Observed, No. & 6 & 13 & 21 & 4 & 44 \\
\hline Expected, No. & 0.79 & 1.08 & 1.98 & 1.55 & 5.40 \\
\hline SIRs & 7.57 & 12.07 & 10.62 & 2.58 & 8.15 \\
\hline $95 \% \mathrm{Cl}$ & $(3.40-16.86)$ & (7.01-20.78) & $(6.92-16.26)$ & $(0.97-6.87)$ & (6.07-10.95) \\
\hline$p$ value & $<0.001$ & $<0.001$ & $<0.001$ & 0.049 & $<0.001$ \\
\hline
\end{tabular}

$\mathrm{Cl}$, confidence interval; MMR, mismatch repair; no., number; SIR, standard incidence ratio.

^One individual with an MLH1 PV was removed from all SIR calculations as she was only 18 at the time of testing and Surveillance, Epidemiology, and End Results incidence rates do not exist for individuals $<20$ years of age. "One individual with a PMS2 PV was removed from the colon cancer SIR calculation as her colon cancer history was ambiguous.

Overall, our cohort of women with MMR PVs more commonly met NCCN BRCA1/2 testing criteria (58.4\%, 243/416) than any of the established LS clinical or testing criteria (Amsterdam II: 23.8\%, 99/416; Revised Bethesda: $22.8 \%, 95 / 416)$.

\section{DISCUSSION}

We describe the reported personal and family cancer histories for a consecutive series of women identified to carry a single germ-line MMR PV through clinical testing with hereditary cancer gene panels. This study is the first, to our knowledge, to provide gene-specific breast cancer risks for all four MMR genes utilizing the same study cohort. In addition, unlike many previous studies, our cohort was not ascertained based on strict LS clinical criteria, suggesting our study population may be more representative of the population undergoing hereditary cancer testing. While many women had a personal or family history of traditional cancers associated with LS (colorectal, endometrial, or ovarian), 11.1\% (46/416) reported no personal or family history of these cancers. In the absence of large panel testing, many might not have been tested for the MMR genes. In addition, three women with breast cancer were found to be positive for a MMR gene PV only after having normal results on a smaller, high-risk breast panel.
We found a twofold and threefold increased risk of breast cancer for the women with MSH6 and PMS2 PVs in our cohort, whereas no breast cancer association was observed for MLH1 or MSH2. PVs in MSH6 and PMS2 were found to confer $31.1 \%$ and $37.7 \%$ cumulative risks for breast cancer by the age of 60 while PVs in MLH1 or MSH2 were found to confer breast cancer risks close to the expected general population risk, $16.1 \%$ and $15.5 \%$, respectively.

Due to differences in study design, cohort ascertainment, and overall proportion of MSH6 and PMS2 PV carriers, many previous studies evaluating breast cancer risk in association with the MMR genes are not suitable for direct comparison with our findings. ${ }^{15,16}$ In one retrospective study with a design more similar to ours, Engel et al. ${ }^{21}$ found an almost twofold increased breast cancer risk in a cohort of 1,107 women with PVs in the MMR genes ( $\mathrm{SIR}=1.9 ; 95 \% \mathrm{CI}, 1.4-2.4)$. Although Engel's study did not include any women with PMS2 PVs and included a smaller proportion of women with MSH6 PVs (16.4\%, C. Engel, personal communication) than the current study (33.1\%), our SIR of 1.96 for all four MMR genes was similar to theirs.

As a clinical laboratory, it was important to determine whether our calculated SIRs for breast cancer might be skewed if our referral population were higher risk than the 


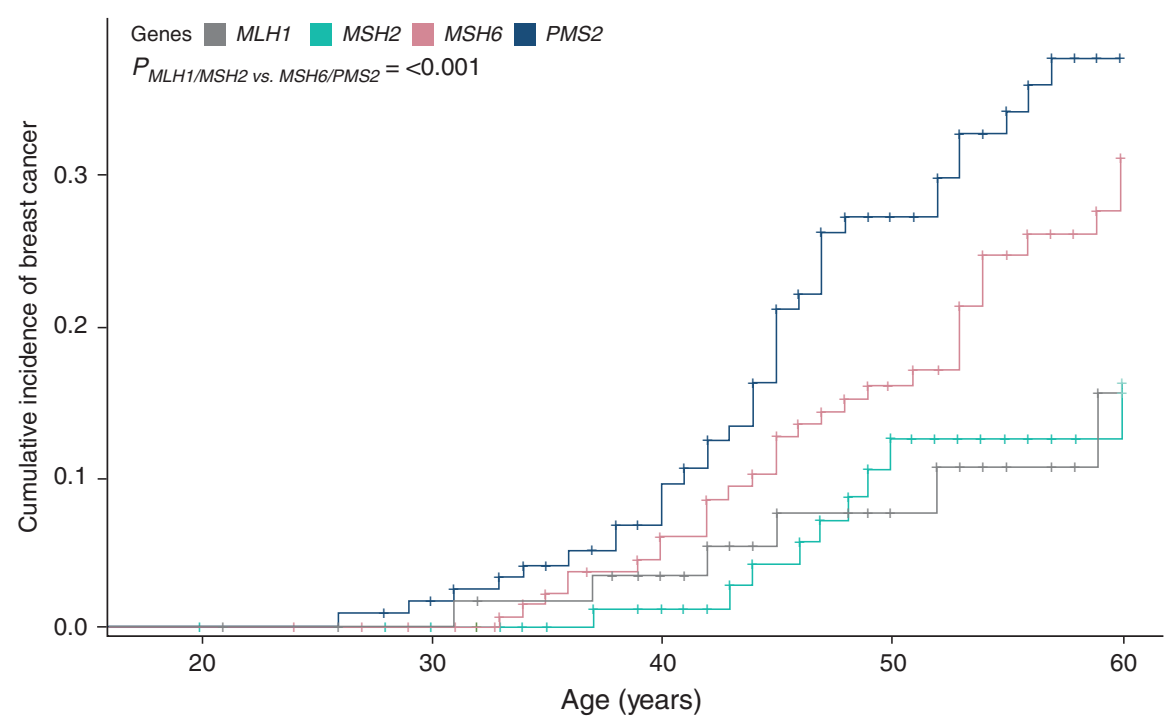

Figure 2 Cumulative incidence of breast cancer. All study participants with breast cancer and a prior cancer diagnosis were assessed to see if there were any potential treatment-related risks that could have contributed to their breast cancer. It was determined that no study participants needed to be excluded based on previous treatment.

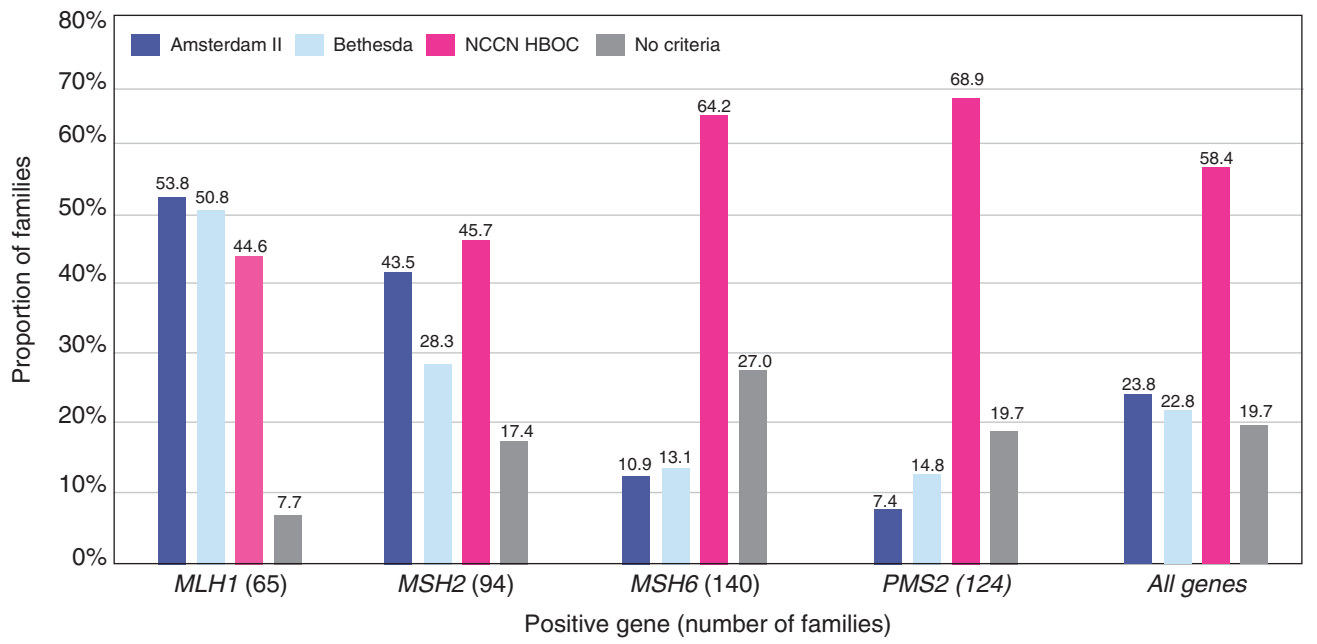

Figure 3 Clinical criteria. Each family is only counted once. Total families $=416$. NCCN HBOC, National Comprehensive Cancer Network Hereditary Breast and Ovarian Cancer.

general population. We therefore calculated SIRs for wellestablished female-specific LS-associated cancers (endometrial and ovarian) using the same cohort of 423 women with MMR PVs. In aggregate, the endometrial cancer risk was found to be 13 -fold higher (SIR $=12.93$; 95\% CI, 10.87-15.37) while the ovarian cancer risk was eightfold higher (SIR $=8.15 ; 95 \%$ CI, 6.61-10.95) compared with the general population, consistent with published LS-associated endometrial $($ SIRs $=10-62)$ and ovarian (SIRs $=7-14$ ) cancer risks. ${ }^{36}$ As LS study cohorts in the literature have historically been ascertained using established clinical and/or testing criteria, resulting in overestimation of published CRC risks, we did not use CRC risks as a metric for comparison. ${ }^{37}$ The fact that the endometrial and ovarian SIRs in our cohort are consistent with previously published SIRs supports the validity of our approach.

Our findings suggest that the current LS clinical testing criteria may not be sufficiently sensitive, particularly for MSH6 and PMS2. Hegde et al. ${ }^{38}$ reported that MSH6 and PMS2 account for approximately $7-10 \%$ and $<5 \%$, respectively, of LS families. When applying Amsterdam II criteria to our cohort, consistent with Hedge et al., only $10.9 \%$ of women with MSH6 and 7.4\% of PMS2 PVs would have been identified. The overall low proportion of women with MMR PVs meeting Amsterdam II Criteria (23.9\%), compared with those meeting NCCN BRCA1/2 testing criteria (58.2\%), underscores the need to continuously reconsider the criteria for which genes to test. 
This study has several limitations. First, the clinical information used in this study was limited to that provided with the testing sample. In addition, the study population consisted only of women considered candidates for genetic testing by their providers and may not be representative of all women with MMR PVs. As our overall testing cohort was comprised largely of women with a personal or family history of breast cancer, SIRs might be biased and will need to be confirmed in other studies. It is notable that the race/ethnicity distribution between this study cohort and the general population differs slightly among certain groups. ${ }^{39}$ To address these potential differences we conducted sensitivity analyses limited to those reported to be only Caucasian/European (non-Hispanic white) in our cohort using breast cancer SEER rates for non-Hispanic white females and found similar results (results not shown). Finally, given our retrospective study design, our estimates of cumulative survival may be overestimated because we may have differentially tested women with longer survival. It is also possible that the retrospective design and young average age at time of testing (52.1 years in this study) underestimates the cumulative incidence. To deal with this latter bias, we estimated cumulative incidence to age 60 only. Prospective validation of our findings will be needed to minimize these and other potential biases.

This study suggests that women with PVs in MSH6 or PMS2 may benefit from increased breast cancer screening. Replication of our gene-specific breast cancer risks will be important to the future development of breast cancer management guidelines for female LS patients. In addition, further assessment of MMR deficiency in breast tumors may provide more information with which to evaluate the link between MMR genes and breast cancer. Finally, family studies of additional individuals with MMR PVs may also provide better breast cancer risk estimates.

In conclusion, our data demonstrate that PVs in MSH6 or PMS2 are associated with a modest but statistically significant increased risk for breast cancer. Previously, the identification of a PV in MSH6 or PMS2 in a woman with breast cancer may have been considered an incidental finding. Here we show that PVs in MSH6 or PMS2 are associated with breast cancer and that these MMR genes should be considered when ordering a multigene panel for women with a personal or family history of breast cancer.

\section{SUPPLEMENTARY MATERIAL}

Supplementary material is linked to the online version of the paper at http://www.nature.com/gim

\section{DISCLOSURE}

M.E.R., S.A.J., L.R.S., M.L.M., A.R.S., B.M., and Z.X. are employed by GeneDx/BioReference Laboratories and have a salary as the only disclosure. W.K.C. is a former employee of BioReference Laboratories. K.S.H., R.T.K., and B.D.S. disclose stock and employment with GeneDx/BioReference Laboratories/Opko. The other authors declare no conflict of interest.

\section{REFERENCES}

1. Lynch HT, Snyder CL, Shaw TG, Heinen CD, Hitchins MP. Milestones of Lynch syndrome: 1895-2015. Nat Rev Cancer. 2015;15:181-194.

2. Castellsagué $E$, Foulkes WD. Lynch syndrome: five unanswered questions. Clin Genet. 2015;87:503-506.

3. Risinger JI, Barrett JC, Watson P, Lynch HT, Boyd J. Molecular genetic evidence of the occurrence of breast cancer as an integral tumor in patients with the hereditary nonpolyposis colorectal carcinoma syndrome. Cancer. 1996;77:1836-1843.

4. Anbazhagan R, Fujii $H$, Gabrielson E. Microsatellite instability is uncommon in breast cancer. Clin Cancer Res. 1999;5:839-844.

5. Blokhuis MM, Goldberg PA, Pietersen GE, et al. The extracolonic cancer spectrum in females with the common "South African" hMLH1 c. C1528T mutation. Fam Cancer. 2008;7:191-198.

6. Shanley S, Fung C, Milliken J, et al. Breast cancer immunohistochemistry can be useful in triage of some HNPCC families. Fam Cancer. 2009;8: 251-255.

7. Jensen UB, Sunde L, Timshel S, et al. Mismatch repair defective breast cancer in the hereditary nonpolyposis colorectal cancer syndrome. Breast Cancer Res Treat. 2010;120:777-782.

8. Walsh MD, Buchanan DD, Cummings MC, et al. Lynch syndromeassociated breast cancers: clinicopathologic characteristics of a case series from the colon cancer family registry. Clin Cancer Res. 2010;16: 2214-2224.

9. Buerki N, Gautier L, Kovac M, et al. Evidence for breast cancer as an integral part of Lynch syndrome. Genes Chromosomes Cancer. 2012;51: 83-91.

10. Lotsari JE, Gylling A, Abdel-Rahman WM, et al. Breast carcinoma and Lynch syndrome: molecular analysis of tumors arising in mutation carriers, non-carriers, and sporadic cases. Breast Cancer Res. 2012;14:R90.

11. Wen $Y H$, Brogi $E$, Zeng $Z$, et al. DNA mismatch repair deficiency in breast carcinoma: a pilot study of triple-negative and non-triplenegative tumors. Am J Surg Pathol. 2012;36:1700-1708.

12. Müller A, Edmonston TB, Corao DA, et al. Exclusion of breast cancer as an integral tumor of hereditary nonpolyposis colorectal cancer. Cancer Res. 2002;62:1014-1019.

13. de Leeuw WJF, van Puijenbroek M, Tollenaar RAEM, Cornelisse CJ, Vasen HFA, Morreau H. Correspondence re: A. Müller et al., Exclusion of breast cancer as an integral tumor of hereditary nonpolyposis colorectal cancer. Cancer Res., 62: 1014-1019, 2002. Cancer Res. 2003;63: 1148-1149.

14. Grandval P, Barouk-Simonet E, Bronner M, et al. Is the controversy on breast cancer as part of the Lynch-related tumor spectrum still open? Fam Cancer. 2012;11:681-683.

15. Pande M, Wei C, Chen J, et al. Cancer spectrum in DNA mismatch repair gene mutation carriers: results from a hospital based Lynch syndrome registry. Fam Cancer. 2012;11:441-447.

16. Win AK, Young JP, Lindor NM, et al. Colorectal and other cancer risks for carriers and noncarriers from families with a DNA mismatch repair gene mutation: a prospective cohort study. I Clin Oncol 2012;30: 958-964.

17. Aarnio M, Sankila R, Pukkala E, et al. Cancer risk in mutation carriers of DNA-mismatch-repair genes. Int J Cancer. 1999;81:214-218.

18. Scott RJ, McPhillips M, Meldrum CJ, et al. Hereditary nonpolyposis colorectal cancer in 95 families: differences and similarities between mutation-positive and mutation-negative kindreds. Am J Hum Genet. 2001;68:118-127.

19. Vasen HF, Morreau H, Nortier JW. Is breast cancer part of the tumor spectrum of hereditary nonpolyposis colorectal cancer? Am J Hum Genet. 2001;68:1533-1535.

20. Dowty JG, Win AK, Buchanan DD, et al. Cancer risks for MLH1 and MSH2 mutation carriers. Hum Mutat. 2013;34:490-497.

21. Engel C, Loeffler M, Steinke $V$, et al. Risks of less common cancers in proven mutation carriers with lynch syndrome. J Clin Oncol. 2012;30: 4409-4415.

22. Harkness EF, Barrow E, Newton K, et al. Lynch syndrome caused by MLH1 mutations is associated with an increased risk of breast cancer: a cohort study. J Med Genet. 2015;52:553-556.

23. Barrow $E$, Robinson L, Alduaij W, et al. Cumulative lifetime incidence of extracolonic cancers in Lynch syndrome: a report of 121 families with proven mutations. Clin Genet. 2009;75:141-149.

24. Richards S, Aziz N, Bale S, et al. Standards and guidelines for the interpretation of sequence variants: a joint consensus recommendation 
of the American College of Medical Genetics and Genomics and the Association for Molecular Pathology. Genet Med. 2015;17:405-424.

25. Kent WJ, Sugnet CW, Furey TS, et al. The human genome browser at UCSC. Genome Res. 2002;12:996-1006.

26. Li H, Durbin R. Fast and accurate short read alignment with BurrowsWheeler transform. Bioinformatics. 2009;25:1754-1760.

27. Van der Auwera GA, Carneiro MO, Hartl C, et al. From FastQ data to high confidence variant calls: the Genome Analysis Toolkit best practices pipeline. Curr Protoc Bioinformatics. 2013;43:11.10.1-33.

28. Li H, Handsaker B, Wysoker A, et al. The Sequence Alignment/Map format and SAMtools. Bioinformatics. 2009;25:2078-2079.

29. Surveillance, Epidemiology, and End Results (SEER) Program. SEER*Stat Database: Incidence-SEER 18 Regs Research Data, Nov 2015 Sub (2000-2013) <Katrina/Rita Population Adjustment> Linked To County Attributes-Total U.S., 1969-2014 Counties, National Cancer Institute, DCCPS, Surveillance Research Program, Surveillance Systems Branch, released April 2016, based on the November 2015 submission www.seer.cancer.gov.

30. Surveillance Research Program. National Cancer Institute SEER*Stat software (version 8.3.2) seer.cancer.gov/seerstat.

31. RStudio Team. RStudio: Integrated Development for R. RStudio: Boston, MA, 2015 http://www.rstudio.com/.

32. Allignol A, Schumacher M, Beyersmann J. Empirical Transition Matrix of Multi-State Models: the etm Package. J Stat Softw. 2011;38:15.

33. Vasen HF, Watson P, Mecklin JP, Lynch HT. New clinical criteria for hereditary nonpolyposis colorectal cancer (HNPCC, Lynch syndrome) proposed by the International Collaborative group on HNPCC. Gastroenterology. 1999;116:1453-1456.

34. Umar A, Boland CR, Terdiman JP, et al. Revised Bethesda Guidelines for hereditary nonpolyposis colorectal cancer (Lynch syndrome) and microsatellite instability. J Natl Cancer Inst. 2004;96:261-268.

35. National Comprehensive Cancer Network. Genetic/Familial High-Risk Assessment: Breast and Ovarian (version 2.2016).
36. Dębniak T, Gromowski T, Scott RJ, et al. Management of ovarian and endometrial cancers in women belonging to HNPCC carrier families: review of the literature and results of cancer risk assessment in Polish HNPCC families. Hered Cancer Clin Pract. 2015;13:3.

37. Carayol J, Khlat M, Maccario J, Bonaïti-Pellié C. Hereditary non-polyposis colorectal cancer: current risks of colorectal cancer largely overestimated. J Med Genet. 2002;39:335-339.

38. Hegde M, Ferber M, Mao R, Samowitz W, Ganguly A; Working Group of the American College of Medical Genetics and Genomics (ACMG) Laboratory Quality Assurance Committee. ACMG technical standards and guidelines for genetic testing for inherited colorectal cancer (Lynch syndrome, familial adenomatous polyposis, and MYH-associated polyposis). Genet Med. 2014;16:101-116.

39. US Census Bureau. Comparative Demographic Estimates, 2016 American Community Survey 1-Year Estimates. 2016. https://factfinder.census.gov/ faces/tableservices/jsf/pages/productview.xhtml?pid = ACS_16_1YR_CP05 \&prodType = table. Accessed 14 September 2017.

This work is licensed under a Creative Commons Attribution 4.0 International License. The images or other third party material in this article are included in the article's Creative Commons license, unless indicated otherwise in the credit line; if the material is not included under the Creative Commons license, users will need to obtain permission from the license holder to reproduce the material. To view a copy of this license, visit http://creativecommons.org/licenses/by/4.0/

(C) The Author(s) 2018 